\title{
Physical and Numerical Contributions to the Structure of Kelvin Wave-CISK Modes in a Spectral Transform Model
}

\author{
AdRian J. MATTHEWS* AND JASON LANDER \\ Department of Meteorology, University of Reading, Reading, Berkshire, United Kingdom
}

(Manuscript received 27 February 1998, in final form 29 December 1998)

\begin{abstract}
"Nonlinear Kelvin wave-CISK modes" are critically assessed as a possible mechanism for the Madden-Julian Oscillation (MJO) with a global spectral transform model and a one-dimensional analog. Convection is parameterized using a simple "positive-only CISK" scheme, where tropospheric diabatic heating is proportional to the low-level convergence, and is set to zero in regions of low-level divergence. Although the modes have many properties that are consistent with the MJO, they also have a serious drawback. The growth rate of unstable modes depends crucially on the width of the heating region, which is resolution dependent. The "CISK catastrophe" has not been averted, and the heating region collapses to the smallest localized scale that the model can support. This scale is larger than the model resolution, as measured by both the gridpoint scale and the inverse wavenumber or half-zonal-wavelength of the highest wavenumber basis function, and is associated with the appearance of negative Gibbs fringes, which are then cut off by the positive-only CISK parameterization.
\end{abstract}

\section{Introduction}

The Madden-Julian Oscillation (MJO) [see Madden and Julian (1994) for a review] has been well documented as a dominant mode of tropical intraseasonal variability. In its simplest form, it consists of planetaryscale tropical convective and associated circulation anomalies that propagate eastward at a phase speed of approximately $5 \mathrm{~m} \mathrm{~s}^{-1}$ over the Eastern Hemisphere. Although the convective anomalies weaken substantially past the date line, the circulation anomalies continue to propagate eastward at a faster phase speed of $15 \mathrm{~m} \mathrm{~s}^{-1}$ over the Western Hemisphere such that the MJO has an overall period of between 30 and 60 days.

Kelvin waves are eastward propagating members of a family of equatorially trapped waves (Matsuno 1966), and have been hypothesized as a likely mechanism for the MJO. The vertical structure of the observed MJO is that of a first-internal tropospheric mode, with opposite sign between the upper- and lower-tropospheric anomalies. However, a dry Kelvin wave with a first-internal mode vertical structure has a phase speed of about $40 \mathrm{~m}$

\footnotetext{
* Current affiliation: School of Environmental Sciences, University of East Anglia, Norwich, United Kingdom.

Corresponding author address: Dr. Adrian J. Matthews, School of Environmental Sciences, University of East Anglia, Norwich, NR4 7TJ United Kingdom.

E-mail: a.j.matthews@uea.ac.uk
}

$\mathrm{s}^{-1}$, much faster than the observed phase speed of the MJO. Diabatic processes, such as "cumulus friction" and Newtonian cooling (Chang 1977), and reduction of static stability by latent heating (Gill 1982), have been invoked to slow down the phase speed of the dry Kelvin wave to one more representative of the observed MJO.

In particular, much attention has been devoted to the "Kelvin wave-CISK" model (e.g., Hayashi and Sumi 1986). This is designed to represent the process of conditional instability of the second kind (CISK), an interaction between convection and large-scale dynamics where deep convection is triggered over a region of lowlevel (moisture) convergence in a conditionally unstable atmosphere. The latent heating associated with the convection forces deep ascending motion, which amplifies the low-level convergence and completes a feedback loop. In its simplest form, a cumulus parameterization is constructed such that tropospheric diabatic heating is defined to be proportional to low-level convergence (e.g., Lau and Peng 1987). In regions of low-level divergence the diabatic heating is set to zero. The vertical structure of the heating is predetermined, usually based upon observations of deep convective heating. When the low-level convergence is due to an atmospheric wave, the process is termed wave-CISK (Hayashi 1970; Lindzen 1974). The strength of the parameterized heating is governed by a heating parameter. For small values of this heating parameter, the system is stable and the Kelvin modes decay. For larger values of the heating parameter, above a certain threshold or critical value, unstable growing nonlinear Kelvin wave-CISK modes 
emerge (Hendon 1988; Bladé and Hartmann 1993). If, in addition, tropospheric diabatic cooling is allowed above regions of low-level divergence, the problem is the analytically simpler one of "linear wave-CISK." Inviscid Kelvin wave-CISK modes tend to have phase speeds of around $10-25 \mathrm{~m} \mathrm{~s}^{-1}$, still faster than the observed phase speed of the MJO. There is also recent observational evidence for convectively coupled Kelvin waves with phase speeds of $10-20 \mathrm{~m} \mathrm{~s}^{-1}$ (e.g., Dunkerton and Crum 1995; Wheeler and Kiladis 1999) that are distinct from the MJO. Kelvin wave-CISK theory may also be relevant to these waves.

Recent modifications to the basic Kelvin wave-CISK model have improved its representation of the MJO. Bladé and Hartmann (1993) incorporated a longitudinally varying basic state such that the mode was unstable and grew over the Eastern Hemisphere, and was stable and decayed over the Western Hemisphere. If frictional convergence in the boundary layer is included, the lowest zonal wavenumbers are destabilized and the phase speed slows to $6 \mathrm{~m} \mathrm{~s}^{-1}$ (Wang 1988; Salby et al. 1994), in line with observations. Lim et al. (1991) and Shen et al. (1996) investigated the effect of vertical wind shear on Kelvin wave-CISK modes and concluded that an easterly vertical shear, as found in the tropical Eastern Hemisphere, tended to destabilize the modes, whereas westerly shear, as found in the Western Hemisphere, tended to stabilize them. This is in agreement with observations of the MJO.

Another feature of a numerical Kelvin wave-CISK mode that can be compared with observations is the dominant spatial scale(s) of the mode, in particular the zonal width of the region of low-level convergence and diabatic heating. Linear wave-CISK theory predicts the largest growth rate at infinitesimally small scale (Hayashi 1970; Crum and Stevens 1983). This "CISK catastrophe" appeared to have been averted when nonlinear or "positive-only" CISK was employed. Hayashi and Sumi (1986) found an eastward propagating 30-day equatorial mode in an integration of a spectral General Circulation Model (GCM) with a Kuo cumulus parameterization scheme. The Kuo scheme is also closed on (moisture) convergence, but it is more sophisticated and realistic than the simple "positive-only" parameterization described above, with extra constraints based on conditional instability of the column and a threshold relative humidity in the cloud layer.

Hayashi and Sumi noted the importance of two scales. The circulation anomalies were of planetary extent (zonal wavenumber one), in agreement with the observed MJO; this feature was central to the acceptance of positive-only wave-CISK as a mechanism for the MJO. A second scale was the width of the heating region, the region of low-level convergence where the positive-only CISK parameterization of deep convective heating was activated. This region had a width of $O(1000 \mathrm{~km})$, well above the grid-scale, and was associated with an organization of convection at the "supercluster" scale.
These two scales were also described in further spectral model studies, using the simple positive-only heating parameterization described above (Lau and Peng 1987; Lau et al. 1989; Bladé and Hartmann 1993; Shen et al. 1996).

However, analytic studies of nonlinear wave-CISK show that the largest growth rates are still associated with infinitesimally small spatial scales: the CISK catastrophe has not been averted by positive-only, or nonlinear, heating (Dunkerton and Crum 1991; Crum and Dunkerton 1992).

This study further investigates nonlinear Kelvin wave-CISK modes in a global spectral transform model and a one-dimensional analog. Particular emphasis is placed on the factors determining the growth rate, and on resolving the apparently paradoxical situation between the finite supercluster scale of the heating region, and the tendency of CISK to collapse to the smallest possible scale.

The global spectral transform model and the initial conditions are briefly described in section 2 , followed by a description of the nonlinear Kelvin wave-CISK modes in section 3, with particular emphasis on the growth rates and width of the heating region. The collapse of the heating region is examined further using a one-dimensional analog model in section 4 , and finally a discussion is presented in section 5 .

\section{Model description}

The model used was the spectral transform, primitive equation model of Hoskins and Simmons (1975). It was run with five sigma levels in the vertical (at $\sigma=0.1$, $0.3,0.5,0.7,0.9)$, and at triangular truncation $M=21$ (T21) in the horizontal. The transform grid had $J_{x}=$ $3 M+1=64$ longitudinal points and $J_{y}=(3 M+1) / 2$ $=32$ Gaussian latitudes, to prevent aliasing of quadratic terms. The only diabatic processes were a $\nabla^{8}$ hyperdiffusion, operating in spectral space with a timescale of $3 \mathrm{~h}$ on the highest total wavenumber, and the convective parameterization operating in gridpoint space, which followed the positive-only CISK scheme of Lau and Peng (1987):

$$
H= \begin{cases}m L q_{\mathrm{bl}} \eta(\sigma) \Delta \sigma D_{\mathrm{bl}} ; & D_{\mathrm{bl}}<0 \\ 0 ; & D_{\mathrm{bl}} \geq 0,\end{cases}
$$

where $H$ is the diabatic heating, $m$ is a variable "moisture availability" or heating factor, $L=2.5 \times 10^{6} \mathrm{~J}$ $\mathrm{kg}^{-1}$ is the latent heat of vaporization, $q_{\mathrm{bl}}=0.02$ is the specific humidity in the boundary layer, and $\eta(\sigma)$ is the prescribed vertical profile of the heating, normalized such that

$$
\sum_{i=1}^{5} \eta\left(\sigma_{i}\right) \Delta \sigma=1,
$$

where $\Delta \sigma$ is the layer thickness, and $D_{\mathrm{bl}}$ is the divergence in the boundary layer, taken as the lowest model 


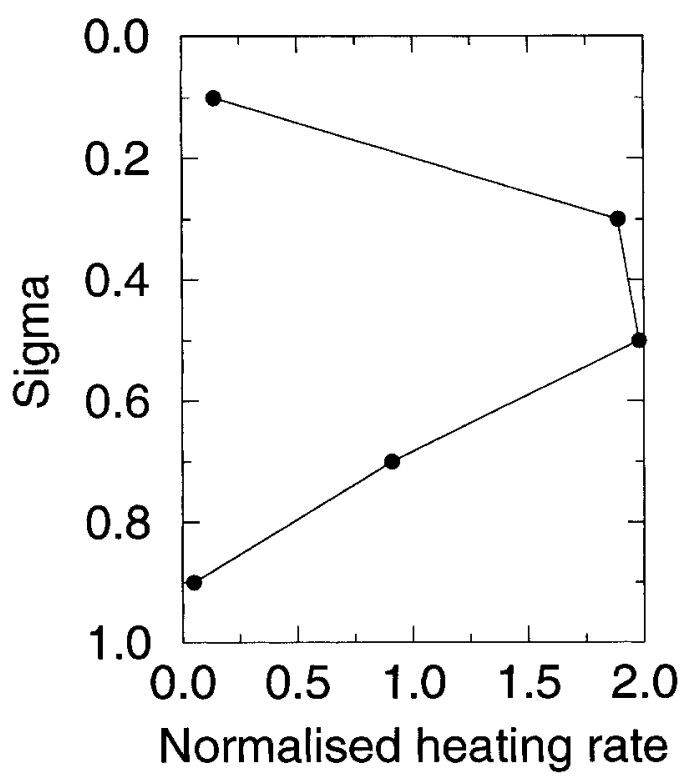

FIG. 1. Heating profile used in the model. The normalized heating rates are in ${ }^{\circ} \mathrm{C}$ day ${ }^{-1}$.

layer $(\sigma=0.9)$. The vertical profile of the heating (Fig. 1 ) was based on observations of the vertical distribution of latent heat release in tropical cumulonimbus clouds during the GARP (Global Atlantic Research Program) Atlantic Tropical Experiment and was the same as that used in Lau and Peng (1987). It peaks in the middle to upper troposphere.

The model was linearized about a resting atmosphere, and initialized with a "dry" Kelvin wave normal mode (Fig. 2). This normal mode was calculated as an eigenvector of the linear tendency matrix of the adiabatic model (without the CISK parameterization). It had a zonal-wavenumber one and first-internal tropospheric mode structure, with an eastward phase speed of $37 \mathrm{~m}$ $\mathrm{s}^{-1}$. The meridional wind field was negligible and the zonal wind anomalies were in phase with geopotential height anomalies (Fig. 2a), indicative of a Kelvin wave structure. The eastward propagation of this dry Kelvin wave can be visualized by considering the phase relationship between the vertical motion field (Fig. 2b) and the temperature field (Fig. $2 \mathrm{c}$ ) at $2.8^{\circ} \mathrm{N}$, the closest latitudinal grid point to the equator. The two are in quadrature, with the midlevel ascent (low-level convergence and upper-level divergence) maximum a quarter wavelength to the east of the temperature minimum. There is no diabatic heating, and midlevel ascent is balanced by adiabatic cooling, hence the temperature structure moves eastward.

\section{Nonlinear Kelvin wave-CISK modes \\ a. Structure}

This initial zonal-wavenumber one Kelvin wave normal mode was superimposed onto the resting atmosphere and the model was integrated forward in time, with the positive-only CISK heating parameterization switched on. A critical value of the heating parameter $\left(m=m_{\mathrm{c}}\right)$ was found to divide a stable and unstable regime, the magnitude of which is sensitive to the ver-

Kelvin Wave Normal Mode

(a) Geopotential Height, Vector Wind $(300 \mathrm{mb})$

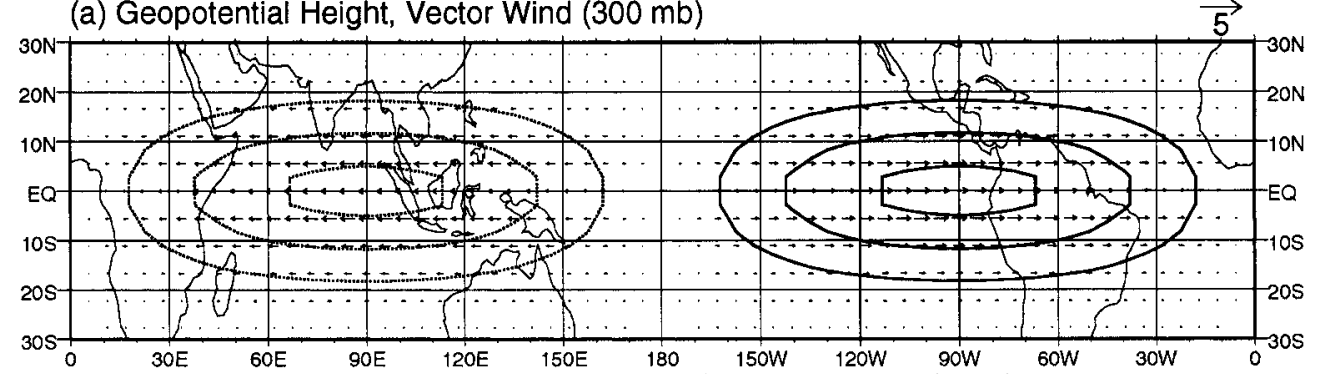

(b) Pressure Velocity $(2.8 \mathrm{~N})$

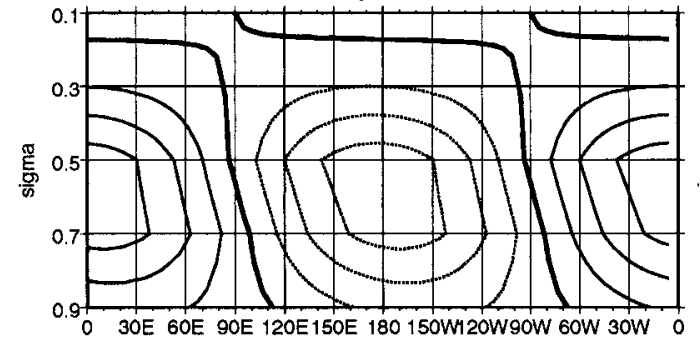

(c) Temperature $(2.8 \mathrm{~N})$



FIG. 2. Structure of initial Kelvin wave normal mode: (a) 300-mb geopotential height anomalies and wind vectors; contour interval is $2 \mathrm{~m}$; negative contours are dotted and the zero contour is omitted; reference wind vector is $5 \mathrm{~m}$ $\mathrm{s}^{-1}$. Longitude-height cross sections at $2.8^{\circ} \mathrm{N}$ of (b) vertical pressure velocity (omega), contour interval is $0.05 \mathrm{mb}$ $\mathrm{h}^{-1}$; (c) temperature anomaly, contour interval is $0.1^{\circ} \mathrm{C}$, and negative contours are dotted. 
(a) Divergence $(2.8 \mathrm{~N})$



(c) Geopotential Height, Vector Wind (300 mb)
Unstable Kelvin Wave-CISK Mode

(b) Temperature $(2.8 \mathrm{~N})$



$\overrightarrow{10}$

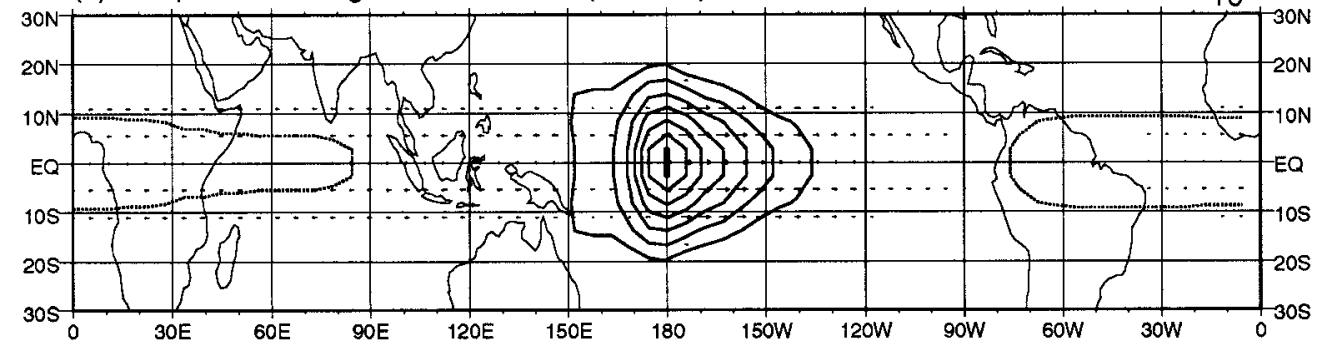

FIG. 3. Structure of unstable Kelvin wave-CISK mode $(m=0.5)$. Longitude-height cross sections at $2.8^{\circ} \mathrm{N}$ of (a) divergence, contour interval is $1 \times 10^{-6} \mathrm{~s}^{-1}$; (b) temperature anomaly, contour interval is $0.2^{\circ} \mathrm{C}$. (c) The $300-\mathrm{mb}$ geopotential height and wind vector anomalies; contour interval is $1.5 \mathrm{~m}$ and reference wind vector is $10 \mathrm{~m} \mathrm{~s}^{-1}$. Negative contours are dashed and the zero contour is omitted. tical heating profile (Chang and Lim 1988). For the vertical heating profile (Fig. 1) and reference temperature profile used in these experiments $m_{\mathrm{c}} \approx 0.38$. Unstable, exponentially growing modes were found for supercritical values of the heating parameter. The model integration, and structure of the unstable mode, for a supercritical value of $m=0.5$ is described below.

The region of low-level convergence, which initially spanned $180^{\circ}$ long, activated the positive-only CISK parameterization and broke up into several smaller-scale regions, which all grew in amplitude. However, the convergence region furthest east grew at a faster rate than the others, and eventually dominated the planetary structure, such that by about day 30 there was a welldefined Kelvin wave-CISK mode, which preserved its shape as it propagated eastward at a constant phase speed of $24 \mathrm{~m} \mathrm{~s}^{-1}$, and whose amplitude grew exponentially with time.

Figure 3 shows the structure of this unstable Kelvin wave-CISK mode, familiar from previous studies (e.g., Lau and Peng 1987). The single narrow region of lowlevel convergence, upper-level divergence, and implied midtropospheric ascent at $180^{\circ}$ in Fig. 3a corresponds to the diabatic heating region. Figure $3 \mathrm{~b}$ shows the temperature structure of the mode. In contrast to the dry Kelvin wave of Fig. 2, the largest positive temperature anomaly was in the heating region itself, at the level of maximum heating. Hence, there was a positive correlation between temperature and diabatic heating, and therefore a generation of eddy available potential energy (which was converted to eddy kinetic energy by the thermally direct circulation), consistent with a growing mode.

Figure $3 \mathrm{c}$ shows the $300-\mathrm{mb}$ geopotential height and wind vector anomalies. The wind is predominantly zonal, indicating the Kelvin wave structure of the mode, with westerlies to the east of the heating region and easterlies to the west, and the opposite-signed structure at $900 \mathrm{mb}$ (not shown). Positive geopotential height anomalies coincide with westerly wind anomalies and negative geopotential height anomalies with easterly wind anomalies. Away from the heating region the mode structure is that of a dry Kelvin wave, with zero potential vorticity anomaly (not shown).

The wind and geopotential height anomalies were much stronger to the east of the heating region, than to the west. This asymmetry can be interpreted as a consequence of the instability of the mode. For the purpose of argument, the Kelvin wave-CISK mode can be regarded as being composed of a diabatic heat source, moving eastward at a reduced speed of $24 \mathrm{~m} \mathrm{~s}^{-1}$, together with the response to that heat source. This reduction in phase speed from that of the deep dry mode has been explained in previous studies by the coalescence of two vertical modes (a shallow and a deep mode) into a single unstable vertical mode (Lau and Peng 1987; Chang and Lim 1988). The low-level convergence is mainly controlled by the shallow vertical mode, which has a slower phase speed, while the deep vertical mode determines the structure of the unstable mode.

The heating forces a Kelvin wave response, which propagates eastward as a fast dry Kelvin wave front (at 
$37 \mathrm{~m} \mathrm{~s}^{-1}$ ) outside the heating region. If the strength of the heat source was constant, this Kelvin wave front would eventually propagate around the globe and impinge on the heating region from the west. However, as the mode is growing, the strength of the heat source increases with time. Hence, a continuous succession of ever stronger wave fronts propagates out at a fast, eastward speed from the heating region. The cumulative effect of these wave fronts is a strong Kelvin response to the east of the heating, that decays with distance from the heating region.

The choice of initial condition was not crucial in obtaining the unstable Kelvin wave-CISK mode. Other Kelvin wave initial conditions (different vertical modes and zonal wavenumbers) also gave rise to the same Kelvin wave-CISK mode.

\section{b. Growth rate}

The growth rate of the Kelvin wave-CISK mode was dependent on the heating parameter $m$. As the heating strength was increased from $m=0$, the diabatic heating partially offset the adiabatic cooling due to ascent, and eventually canceled it at the critical value $m=m_{\mathrm{c}}$. As ascent and adiabatic cooling are proportional to lowlevel convergence through mass conservation, and the diabatic heating was defined to be proportional to the low-level convergence [Eq. (1)], the relative offset of the adiabatic cooling by the diabatic heating was independent of the actual magnitude of the low-level convergence.

As the heating strength was increased further $(m>$ $m_{\mathrm{c}}$ ), the diabatic heating became stronger than the adiabatic cooling. A positive temperature anomaly developed in the heating region, and an exponentially growing, unstable Kelvin wave-CISK mode developed. The solid line in Fig. 4 shows growth rates for T21 Kelvin wave-CISK modes for a range of values of the heating parameter $m$. For supercritical values of $m$, the growth rate $\gamma$ was approximately proportional to $m-m_{\mathrm{c}}$ :

$$
\gamma \approx a\left(m-m_{\mathrm{c}}\right) \text { if } m>m_{\mathrm{c}} .
$$

The phase speed of the unstable Kelvin wave-CISK mode decreased only slightly with increasing $m$ above the critical value ( $23 \mathrm{~m} \mathrm{~s}^{-1}$ at $\left.m=0.7\right)$, consistent with the phase speed being determined by the coalescence of two vertical modes (Lau and Peng 1987).

For subcritical values of the heating parameter $(m<$ $m_{\mathrm{c}}$ ), the resulting moist Kelvin wave structures were stable and slowly decayed. It is also apparent from Fig. 4 that these stable moist Kelvin wave structures decayed slightly faster with stronger heating. This can be traced to the existence of a negative temperature anomaly in the heating region, leading to a negative correlation between temperature and diabatic heating and hence the destruction of eddy available potential energy, and a decaying mode. A larger value of the heating parameter $m$, as long as it remains subcritical, will lead to a greater



FIG. 4. Growth rates of Kelvin wave-CISK modes. T21 (T42) integrations are shown by the solid (dotted) lines.

rate of destruction of eddy available potential energy, and therefore to a more strongly decaying mode. The stable moist Kelvin wave structures that arose from integrations with subcritical heating parameters were rather sensitive to initial conditions and are not discussed further.

The experiments described above were repeated with a doubled horizontal resolution (T42). A Kelvin waveCISK mode emerged with essentially the same largescale structure and phase speed as in the T21 experiments, and $m_{\mathrm{c}} \approx 0.38$ was unchanged. However, the growth rates of the T42 modes were higher than those of the T21 modes, for the same value of the heating parameter $m$ (Fig. 4, dotted line). The gradient of the line (a) has approximately doubled from the T21 to T42 integrations. This appears to be due to a change in the width of the heating region, which has approximately halved in the T42 experiments, and is discussed in the next section.

The T42 integrations retained the same vertical resolution and hence the same vertical modes as the T21 integrations. The phase speeds of the nonlinear Kelvin wave-CISK modes remained unchanged between the T21 and T42 experiments, again consistent with the coalescence of vertical modes interpretation.

\section{c. Width of heating region}

Analytic studies of nonlinear wave-CISK show that the largest growth rate occurs at the highest wavenumber, that is, the CISK catastrophe has not been averted by positive-only heating (Dunkerton and Crum 1991; Crum and Dunkerton 1992). In contrast, the collapse of the heating region in the experiments in this study seems to have been halted. The longitudinal half-width of the 
heating region (i.e., from maximum to zero) was approximately

$$
\Delta_{\mathrm{HR}} \approx 1510 \mathrm{~km} \approx 13.6^{\circ}
$$

for the T21 experiments. This appears to be substantially larger than the model resolution, measured by either the longitudinal grid spacing

$$
l_{\mathrm{GRID}}=\frac{2 \pi a}{J_{x}} \approx 625 \mathrm{~km} \approx 5.6^{\circ},
$$

where $a=6370 \mathrm{~km}$ is the radius of the earth and $J_{x}$ is the number of longitudinal grid points, or the inversewavenumber of the highest-wavenumber basis function

$$
l_{\mathrm{T}}=\frac{a}{[M(M+1)]^{1 / 2}} \approx 296 \mathrm{~km} \approx 2.7^{\circ},
$$

where $M$ is the truncation wavenumber, or alternatively half a zonal wavelength of the highest-wavenumber basis function

$$
l_{\mathrm{HZW}}=\frac{\pi a}{M} \approx 953 \mathrm{~km} \approx 8.6^{\circ} .
$$

Previous studies have attributed this finite scale of the heating region to dissipation (Lau and Peng 1987) or to a physical supercluster scale (Lau et al. 1989). However, when the resolution of the model was doubled from T21 to T42, the half-width of the heating region was approximately halved from $\Delta_{\mathrm{HR}} \approx 13.6^{\circ}$ to $\Delta_{\mathrm{HR}} \approx 5.7^{\circ}$, suggesting that it is governed by a numerical, rather than a physical, mechanism.

The unstable modes have a very similar spatial structure at both T21 and T42 resolution, except for the width of the heating region. The increase in growth rate with resolution noted in section $3 \mathrm{~b}$ can be explained in terms of this width. Consider a mode with a change in zonal wind $\Delta u$ occurring across longitudinal half-width $\Delta_{\mathrm{HR}}$. As the diabatic heating is proportional to the convergence in the lowest model level and the meridional wind perturbation is negligible, the heating is approximately proportional to $\Delta u / \Delta_{\mathrm{HR}}$. For any $\Delta u$, the half-width $\Delta_{\mathrm{HR}}$ of the T42 mode is around half that of the T21 mode, implying a doubling of the strength of the heating and a doubling of the growth rate.

The hyperdiffusion in the model selectively damped out the smallest scales and could be responsible for the scale-locking of the width of the heating region. However, if the hyperdiffusion was turned off, the spatial structure of the Kelvin wave-CISK mode remained essentially unchanged. The growth rate increased slightly, and the half-width of the heating region decreased slightly, from $\Delta_{\mathrm{HR}} \approx 13.6^{\circ}$ to $\Delta_{\mathrm{HR}} \approx 11.3^{\circ}$ for $\mathrm{T} 21$ truncation. This is still larger than the grid-spacing and inverse-wavenumber scales quoted above.

On closer inspection, the length-scale comparisons made above are misleading. The inverse wavenumber scales $l_{\mathrm{T}}$ and $l_{\mathrm{HZW}}$ apply to strictly periodically repeating functions that cover the entire global domain. As the heating region has a localized structure, it will have significant Fourier contributions from wavenumbers lower than the truncation limit, therefore its characteristic scale $\Delta_{\mathrm{HR}}$ will be greater than $l_{\mathrm{T}}$ and $l_{\mathrm{HZW}}$. Following Lander and Hoskins [1997; their Eq. (25)], the size of the smallest resolved localized feature in a spectral transform model can be characterized by

$$
\eta_{2 \mathrm{D}} \approx \frac{1.6 \pi a}{[M(M+1)]^{1 / 2}} \approx 1490 \mathrm{~km} \approx 13.4^{\circ},
$$

where $\eta_{2 \mathrm{D}}$ is the distance between the central peak and the first minimum of the (Bessel-like) function formed by truncating the spectral series for a Dirac delta function at wavenumber $M$. As $\Delta_{\mathrm{HR}} \approx \eta_{2 \mathrm{D}}$, it appears that the heating region has collapsed to the smallest possible scale, and the "CISK catastrophe" has not been averted.

It should be clarified at this point what is meant by the "CISK catastrophe" in a model with positive-only CISK heating. The catastrophe of linear CISK is that the largest growth rate is at the largest zonal wavenumber or smallest scale. In this linear framework, this corresponds to a perturbation that is periodically repeated throughout the whole longitudinal domain. In the case of positive-only CISK heating, the fastest growing "mode" also appears to have a heating region at the smallest model scale. However, the heating region is localized and does not repeat itself periodically throughout the domain. The response to this localized heating is global and is dominated by the lowest zonal wavenumbers. This feature explains the success that positiveonly CISK has had in explaining certain features of observed circulations such as the MJO, and is not in question here. What is being questioned is the attribution of a physical significance to the finite width of the heating region.

In the next section, a series of analog one-dimensional experiments are performed to further investigate the numerical mechanisms behind the scale-locking of the width of the heating region.

\section{One-dimensional analog experiments}

In a system as complex as a full spectral transform model, it is difficult to isolate the effect of the truncation from that of the "real" dynamics. In this section, a series of one-dimensional experiments designed as analogs to the "CISK catastrophe" is described.

The one-dimensional model used is described in detail in Lander and Hoskins (1997). In summary, Fourier basis functions are used to represent some physical quantity, denoted by $Q$, on a periodic domain $-L / 2 \leq$ $x \leq L / 2$, where $L=2 \pi a \approx 40000 \mathrm{~km}$ or $L=360^{\circ}$. The parameterization tendencies are evaluated by projecting the spectral representation onto a transform grid, calculating the tendencies on this grid, and then transforming these back into spectral space. Like its counterpart in the full spherical model, the transform grid is 


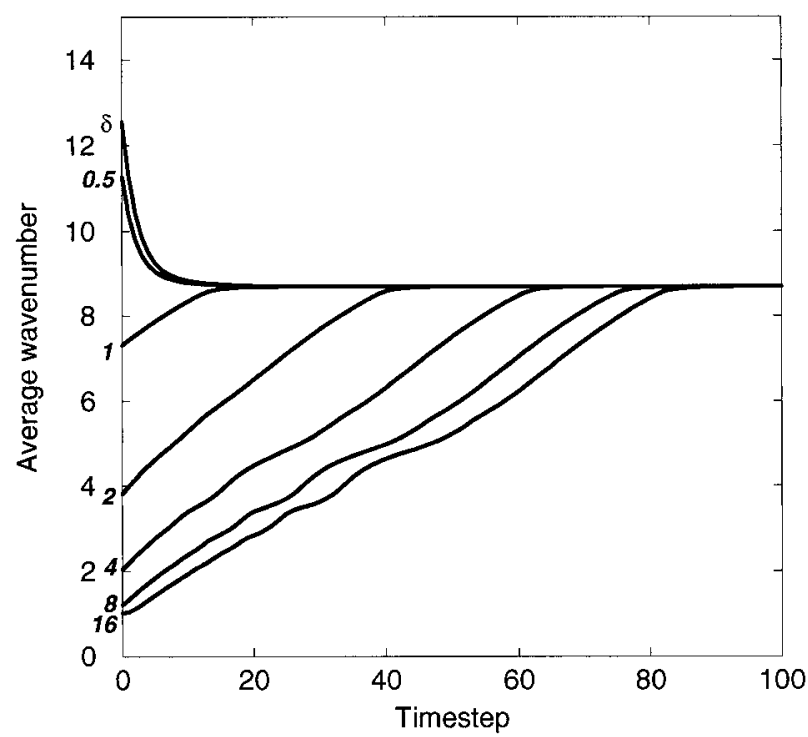

FIG. 5. Average wavenumber as a function of time step for a series of one-dimensional CISK analog experiments. The initial conditions, in order of increasing wavenumber, were Gaussians with scale-widths of $w=16,8,4,2,1$, and 0.5 times the width of a grid box $\left(l_{\text {GRID }}\right)$ and a "delta function."

finer than is required to resolve the smallest basis function, to prevent aliasing.

For comparison with the Kelvin wave-CISK experiments on the sphere in section 3, the one-dimensional analog experiments in this section were truncated at wavenumber $M=21$, and used a transform grid of $J$ $=3 M+1=64$ grid points. The grid spacing is the same as the longitudinal grid spacing in the full spherical model:

$$
l_{\mathrm{GRID}}=\frac{2 \pi a}{J} \approx 625 \mathrm{~km} \approx 5.6^{\circ} .
$$

The only forcing in the model was intended to have similar characteristics to the CISK heating [Eq. (1)] in the full spherical model;

(a) Grid-point values

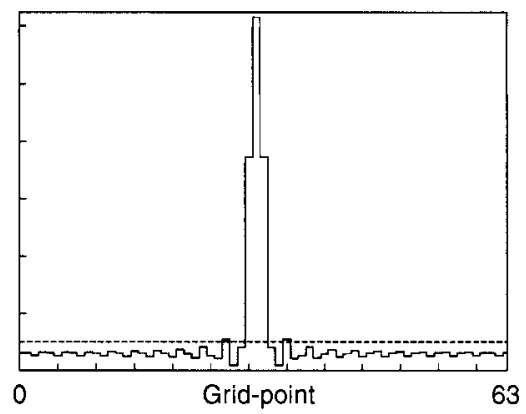

$$
\frac{\partial Q}{\partial t}= \begin{cases}\alpha Q ; & Q>0 \\ 0 ; & Q \leq 0\end{cases}
$$

The analog model was designed to share some characteristics (in particular the scale of the "heating region") with the Kelvin wave-CISK model, rather than actually being a one-dimensional version of it. Here $Q$ can be thought of as a generalized variable representing the amplitude of the Kelvin wave-CISK modal structure at each longitude. The region of positive $Q$ then represents the region of low-level convergence, ascent, and heating in the Kelvin wave-CISK model. The constant $\alpha$ term can be thought of as representing the feedback between low-level convergence and the ascent generated by the heating.

The model was integrated forward from a suitable initial state using a simple forward-difference scheme. At each time step the mean value of $Q$ was also adjusted so that it remained constant with time. Figure 5 shows the evolution of seven such integrations, identical apart from their initial conditions. The initial conditions were defined on the transform grid; the first six consisted of Gaussian profiles of scale-widths $w=16,8,4,2,1$, and $0.5 l_{\text {GRID }}$ (grid points), where a Gaussian was defined in the standard form $\exp \left(-x^{2} / 2 w^{2}\right)$ and the seventh consisted of a nonzero value at a single grid point, with zeroes elsewhere (the equivalent of a delta function). The scale of the field was characterized by its average wavenumber $\bar{k}$, defined as

$$
\bar{k}^{2}=\frac{\sum k^{2} P_{k}^{2}(Q)}{\sum P_{k}^{2}(Q)},
$$

where $\mathcal{P}_{k}^{2}(Q)$ is the power at wavenumber $k$ in the field $Q$. All the initial states either collapsed or expanded to the same scale.

The final $Q$-field that all the initial conditions converged on is shown in Fig. 6a. It can be seen that the "heating region" did not collapse to a single grid point, but was smeared out. Its half-width, as defined in the previous section, was approximately two grid points (b) Power spectrum

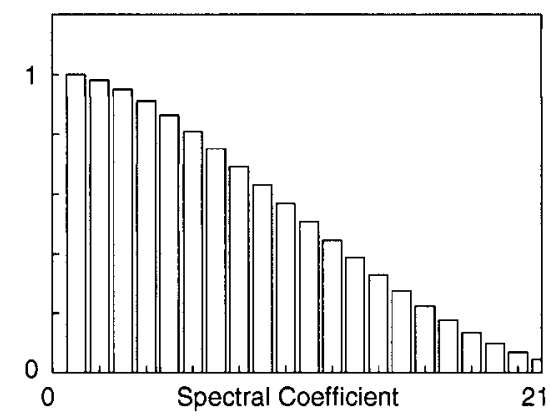

FIG. 6. (a) Structure and (b) power spectrum of the scale-locked state in the one-dimensional CISK analog experiments. 


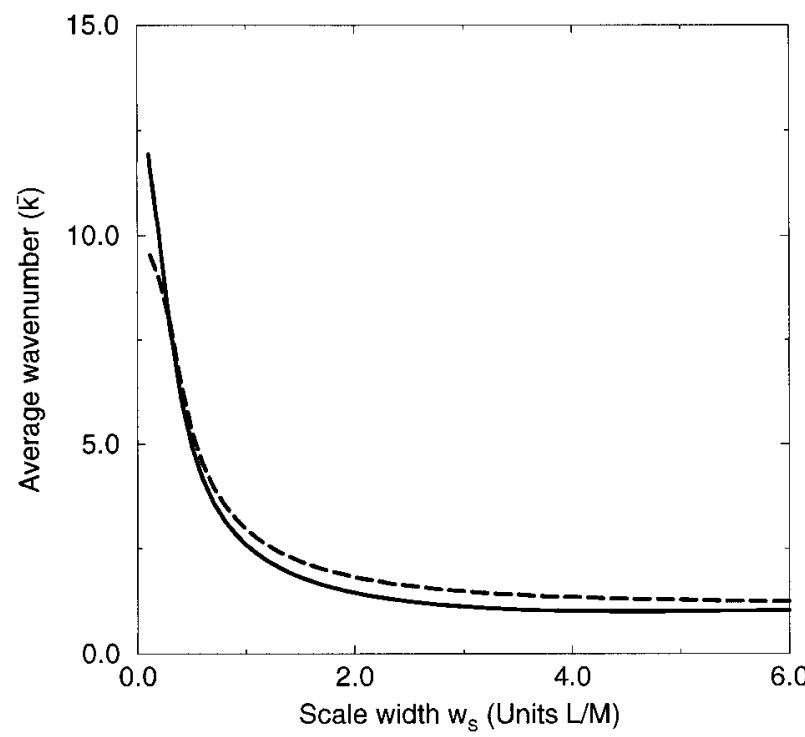

FIG. 7. Average wavenumber of the fields input to (solid line) and output from (dotted line) the CISK analog parameterization as a function of the scale width $w$ of the Gaussian input field. The scale width is measured in units of $L / M$.

$$
\Delta_{\mathrm{HR}} \approx 1250 \mathrm{~km} \approx 11.3^{\circ} \text {. }
$$

This is close to the half-width of the heating region in the Kelvin wave-CISK mode on the sphere in the experiment with no hyperdiffusion. It appears that the greatly simplified one-dimensional analog experiments have captured some of the essential characteristics of the full Kelvin wave-CISK experiments, and reproduced a "heating region" of the same scale. The power spectrum of the final $Q$-field (Fig. 6b) tailed off with wavenumber, and was not constant with wavenumber like that of a delta function showing that the CISK-like scheme has collapsed to a scale just slightly larger than that of the smallest resolvable localized feature.

This scale-locking of the "heating region" appears to be a direct result of poor resolution of the tendencies produced by the parameterization. Figure 7 shows the average wavenumbers of the field used as input to the CISK analog parameterization scheme and of the field produced as output, as a function of the scale of the input data. The input data consisted of a series of Gaussians of varying scale-widths $w$ on a grid with $J=64$ points represented using a spectral series truncated at $M=21$. For all Gaussians with $w>w_{\mathrm{c}}$, where

$$
w_{\mathrm{c}} \approx 0.3 L / M \approx 0.9 l_{\mathrm{GRID}} \approx 560 \mathrm{~km} \approx 5.1^{\circ}
$$

the tendency produced by the parameterization had a higher $\bar{k}$ than the input data: it acted to shrink the feature. In contrast, for $w<w_{\mathrm{c}}$ the tendency had a larger $\bar{k}$ than the input field and acted to expand the feature. Under these circumstances one would expect to see precisely the behavior implied by Fig. 5 where an initial peak collapsed or expanded until the input field and the tendencies were on the same scale.
The scale at which the collapse stopped coincided with the appearance of Gibbs fringes (Fig. 6a), the spurious maxima and minima that appear around a smallscale feature when it is spectrally truncated. These fringes can be thought of as the "penalty" that has to be paid for having such a small-scale feature: the smaller a feature is, the more pronounced the fringes around it become. Turning this argument around: if the fringes were to be removed, as the CISK analog parameterization does by taking only that part of the field where $Q>0$, the result must be larger scale. This explains the behavior seen in Fig. 5.

It is interesting to compare the scale of the Gaussian with critical scale width $w_{\mathrm{c}}$ to the scale of a truncated delta function. Lander and Hoskins (1997) showed that the one-dimensional equivalent of the characteristic scale in Eq. (8) is

$$
\eta_{1 \mathrm{D}} \approx \frac{1.4 \pi a}{M} \approx 1330 \mathrm{~km} \approx 12.0^{\circ} .
$$

Virtually all the area under the truncated delta function is within the region $-\eta_{1 \mathrm{D}}<x<\eta_{1 \mathrm{D}}$. Compare this with the Gaussian where $98 \%$ of the area lies within $-2 w_{\mathrm{c}}<x<2 w_{\mathrm{c}}$. The width of the Gaussian can be therefore be characterized by

$$
2 w_{\mathrm{c}} \approx 1130 \mathrm{~km} \approx 10.1^{\circ},
$$

which is of the order of $\eta_{1 \mathrm{D}}$.

\section{Discussion}

A global spectral transform model and a one-dimensional analog model have been used to examine the properties of nonlinear Kelvin wave-CISK modes, with particular emphasis on the factors that determine the growth rate and the width of the heating region. In the global model, convective heating was simply parameterized to be proportional to low-level convergence, and to be zero in regions of low-level divergence. For supercritical values of the heating parameter $m>m_{\mathrm{c}}$, exponentially growing nonlinear Kelvin wave-CISK modes emerged, whose growth rate was approximately proportional to $m-m_{\mathrm{c}}$.

The growth rate of the Kelvin wave-CISK modes is also highly dependent on the width of the heating region, which is determined by the model resolution. The linear CISK catastrophe, where the largest growth rates are found at the smallest scales, has not been averted by positive-only heating, and the heating region does collapse to the smallest localized scale that the model with its CISK parameterization can support.

However, this scale is larger than may be initially expected, which could explain its previous attribution to a physically meaningful quantity. The positive-only CISK parameterization acts to shrink the region of lowlevel convergence toward the gridpoint scale. However, as it approaches this scale, negative Gibbs fringes start to appear at its flanks. When calculating the heating 
from this field, these fringes are cut off by the positiveonly CISK parameterization, smearing the heating region out. An equilibrium scale is eventually attained where the shrinking tendency of the CISK parameterization is balanced by an expanding tendency from the spectral truncation and the positive-only aspect of the CISK parameterization. It is this numerically determined and resolution-dependent equilibrium scale that has been previously associated with a supercluster scale.

Kelvin wave-CISK theory, using the simple positiveonly convective parameterization, has been successful in explaining many aspects of the observed MJO, including the eastward propagation and planetary scale of the tropical circulation anomalies, and their longitudinal variations. However, the shortcomings of the theory highlighted by this study need to be addressed; the width of the heating region, which has previously been attributed to a supercluster scale and thought to be larger than the smallest scale in the model, is determined entirely by the horizontal resolution of the model; the CISK catastrophe has not been averted, and the heating region has shrunk to its smallest possible scale.

Acknowledgments. The authors thank Brian Hoskins for many helpful discussions during the course of this research. The comments of Matthew Wheeler, George Kiladis, and an anonymous reviewer helped to greatly improve the manuscript from its original version. This work was carried out while the authors were in receipt of NERC Research Studentships at the Department of Meteorology, University of Reading.

\section{REFERENCES}

Bladé, I., and D. L. Hartmann, 1993: Tropical intraseasonal oscillations in a simple nonlinear model. J. Atmos. Sci., 50, 29222939.

Chang, C.-P., 1977: Viscous internal gravity waves and low-frequency oscillations in the Tropics. J. Atmos. Sci., 34, 901-910.

- , and H. Lim, 1988: Kelvin wave-CISK: A possible mechanism for the 30-50 day oscillations. J. Atmos. Sci., 45, 1709-1720.

Crum, F. X., and D. E. Stevens, 1983: A comparison of two cumulus parameterization schemes in a linear model of wave-CISK. $J$. Atmos. Sci., 40, 2671-2688.
- , and T. J. Dunkerton, 1992: Analytic and numerical models of wave-CISK with conditional heating. J. Atmos. Sci., 49, 16931708.

Dunkerton, T. J., and F. X. Crum, 1991: Scale selection and propagation of wave-CISK with conditional heating. J. Meteor. Soc. Japan, 69, 449-458.

, and - 1995: Eastward propagating 2- to 15-day equatorial convection and its relation to the the tropical intraseasonal oscillation. J. Geophys. Res., 100, 25 781-25 790.

Gill, A. E., 1982: Studies of moisture effects in simple atmospheric models: The stable case. Geophys. Astrophys. Fluid Dyn., 19, 119-152.

Hayashi, Y.-Y., 1970: A theory of large-scale equatorial waves generated by condensation heat and accelerating the zonal wind. $J$. Meteor. Soc. Japan, 48, 140-160.

- , and A. Sumi, 1986: The 30-40 day oscillations simulated in an "aqua-planet" model. J. Meteor. Soc. Japan, 64, 451-467.

Hendon, H. H., 1988: A simple model of the 40-50 day oscillation. J. Atmos. Sci., 45, 569-584.

Hoskins, B. J., and A. J. Simmons, 1975: A multi-layer spectral model and the semi-implicit method. Quart. J. Roy. Meteor. Soc., 101, 637-655.

Lander, J., and B. J. Hoskins, 1997: Believable scales and parameterizations in a spectral transform model. Mon. Wea. Rev., 125, 292-303.

Lau, K.-M., and L. Peng, 1987: Origin of low-frequency (intraseasonal) oscillations in the tropical atmosphere. Part I: Basic theory. J. Atmos. Sci., 44, 950-972.

,-- C. H. Sui, and T. Nakazawa, 1989: Dynamics of super cloud clusters, westerly wind bursts, 30-60 day oscillations and ENSO: A unified view. J. Meteor. Soc. Japan, 67, 205-219.

Lim, H., C.-P. Chang, and T. K. Lim, 1991: Vertical wind shear effects on Kelvin wave-CISK modes: Possible relevance to 30-60 day oscillations. Terr. Atmos. Oceanic Sci., 2, 203-215.

Lindzen, R. S., 1974: Wave-CISK in the Tropics. J. Atmos. Sci., 31, 156-179.

Madden, R. A., and P. R. Julian, 1994: Observations of the 40-50 day tropical oscillation-A review. Mon. Wea. Rev., 122, 814837.

Matsuno, T., 1966: Quasi-geostrophic motions in the equatorial area. J. Meteor. Soc. Japan, 44, 25-42.

Salby, M. L., R. R. Garcia, and H. H. Hendon, 1994: Planetary circulations in the presence of climatological and wave-induced heating. J. Atmos. Sci., 51, 2344-2367.

Shen, W., M. A. Geller, and M. Zhang, 1996: Generalization of the effect of vertical shear of mean zonal flow on tropical CISKwave excitation. J. Atmos. Sci., 53, 2166-2185.

Wang, B., 1988: Dynamics of tropical low frequency waves: An analysis of the moist Kelvin wave. J. Atmos. Sci., 45, 20512065.

Wheeler, M., and G. N. Kiladis, 1999: Convectively coupled equatorial waves: Analysis of clouds and temperature in the wavenumber-frequency domain. J. Atmos. Sci., 56, 374-399. 